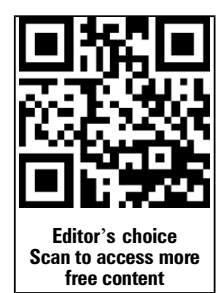
free content

\title{
High levels of indium exposure relate to progressive emphysematous changes: a 9-year longitudinal surveillance of indium workers
}

\author{
Atsuko Amata, ${ }^{1,2}$ Tatsuya Chonan, ${ }^{1}$ Kazuyuki Omae, ${ }^{3}$ Hiroshi Nodera, ${ }^{1}$ Jiro Terada, ${ }^{2}$ \\ Koichiro Tatsumi ${ }^{2}$
}

- Additional material is published online only. To view please visit the journal online (http://dx.doi.org/10.1136/ thoraxjnl-2014-206380)

1 Department of Medicine, Nikko Memorial Hospital, Hitachi, Japan

${ }^{2}$ Department of Respirology, Graduate School of Medicine, Chiba University, Chiba, Japan ${ }^{3}$ Department of Preventive Medicine and Public Health, School of Medicine, Keio University, Tokyo, Japan

\section{Correspondence to} Dr Jiro Terada, Department of Respirology, Graduate School of Medicine, Chiba University, 1-8-1 Inohana Chuoku Chiba city, Chiba 2608670, Japan; jirotera@chiba-u.jp

Received 1 October 2014 Revised 29 June 2015 Accepted 22 July 2015 Published Online First 18 August 2015

\section{ABSTRACT}

Background During the last decade it has been clarified that the inhalation of indium compounds can evoke alveolar proteinosis, cholesterol granuloma, pulmonary fibrosis and emphysema. In this study, we aimed to elucidate the characteristics and time course of pulmonary disorders among indium workers using comprehensive pulmonary examinations at an indiumprocessing factory.

Methods Data for 84 male workers who underwent the examinations for nine consecutive years from 2002 to 2010 were analysed regarding their symptoms, serum indium concentration (sIn), serum markers of interstitial pneumonia, pulmonary function test parameters and high-resolution CT (HRCT) findings of the lungs.

Results In association with improvements in the work environment and work practice, the sln levels decreased with significant reductions in the KL-6 and surfactant protein D (SP-D) levels. Regarding the HRCT findings, the interstitial lesions regressed partially, whereas emphysematous lesions increased progressively in the workers with high sIn values. $\mathrm{FEV}_{1} / \mathrm{FVC}$ decreased with the years and the rate of decrease was significantly greater in those with high sln. The biological half-life of sln was estimated to be 8.09 years.

Conclusions The present findings suggest that the $\sin$, SP-D, KL-6 levels and radiological interstitial changes can be reduced in indium workers by alleviating exposure to indium, whereas emphysematous lesions can progress among those with a history of heavy exposure.

\section{INTRODUCTION}

Indium is a rare metal that is primarily used in the form of indium-tin oxide (ITO), a sintered material consisting of $90 \%$ indium oxide $\left(\mathrm{In}_{2} \mathrm{O}_{3}\right)$ and $10 \%$ tin oxide $\left(\mathrm{SnO}_{2}\right)$, to produce flat panel displays, such as liquid-crystal displays. Recently, indium use has sharply increased; the world demand for indium in 2007 was approximately four times higher than in $1999 .{ }^{1}$

In 2001, a 29-year-old man who was an ITO surface grinder died of interstitial pneumonia with CrossMark

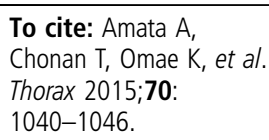

bilateral pneumothorax, ${ }^{2}$ constituting the first reported case of indium-related lung disease. In 2002, we conducted a comprehensive study at the ITO plant where the above patient was employed and reported that interstitial lung disorders characterised by abnormalities on high-resolution CT (HRCT) and the elevation of serum KL-6 were

\section{Key messages}

What is the key question?

- Does the progress of interstitial and/or emphysematous lesion in indium workers change by alleviating exposure to indium?

\section{What is the bottom line?}

- Because permanent emphysematous changes may be evoked under heavy exposure to indium compounds, it is necessary to reduce exposure to indium-containing particles and detect lung disorders caused by indium as early as possible.

\section{Why read on?}

- This comprehensive study, for the first time, examined the same workers in an indium plant for nine consecutive years, showing different longitudinal patterns of interstitial and emphysematous lesions in patients with indium lung after stopping or decreasing the exposure to the inhaled indium compounds.

observed in a significant number of indiumprocessing workers. ${ }^{3}$ Furthermore, workers with serum indium (sIn) concentrations in the highest quartile had a history of significantly longer periods of exposure, greater HRCT changes, lower diffusing capacity of the lung for carbon monoxide $\left(\mathrm{DL}_{\mathrm{CO}}\right)$ and higher KL-6 levels compared with those with sIn concentrations in the lowest quartile. According to these findings, we proposed that inhaled indium compound may be a potential new cause of occupational lung disease ('indium lung'). ${ }^{1}{ }^{3}$ This hypothesis was subsequently confirmed in different populations of indium workers. ${ }^{4}$ Combining the data obtained from 12 factories, including ours, and one research laboratory, Nakano et $a l^{5}$ reported a strong dose-response relationship between the sIn level and effects on the lungs.

As of May 2013, nine cases of interstitial lung disease from Japan ${ }^{6-11}$ and three cases of alveolar proteinosis from the USA ${ }^{12}$ and China ${ }^{13}$ had been reported. Exposure to indium compounds has been suggested to cause a novel lung disease consisting of alveolar proteinosis, cholesterol granuloma, pulmonary fibrosis and emphysema, accompanied by 
pneumothorax in severe cases. ${ }^{14}$ The work environment and practice at ITO plants, where most cases have been reported, have considerably improved, and workplace changes have been implemented among heavily exposed workers over the last decade. In this study, we conducted a longitudinal surveillance for nine consecutive years starting in 2002 of 84 male indium workers at an indium plant, where a cross-sectional study of 108 indium-processing workers was previously conducted, ${ }^{3}$ to elucidate the clinical course of indium lung and the effects of reduced exposure.

\section{MATERIALS AND METHODS}

\section{Study design and subjects}

The present study was conducted as part of the health maintenance programme for the employees and approved by the medical professional committee that oversees the health maintenance programme of the plant.

A comprehensive respiratory examination programme was conducted annually from 2002 to 2010 for workers who were formerly and/or currently engaged in indium processing. The study design has been reported elsewhere and consisted of examinations of signs and symptoms, pulmonary function tests, HRCT of the lungs and measurements of the sIn concentration and serum markers of interstitial lung disease. ${ }^{3}$ Five hundred and fifteen individuals received an examination at least once, among whom 108 male workers underwent examinations in 2002 (reported as a cross-sectional study ${ }^{3}$ ). After excluding the data for 24 subjects who failed to undergo an examination for one or more years, we analysed the data of 84 male workers (figure 1). The exposure period for these workers was 9.7 \pm 4.8 years (table 1 ). During the study period, workers with significant interstitial change on chest X-rays or HRCT, emphysematous changes accompanied by elevated KL-6 and/or SP-D levels, or high sIn concentrations (2002-2006: no criteria, 2007-2008: > $20 \mathrm{ng} / \mathrm{mL}, 2009-$ : >10 ng/mL) were transferred to non-indium sections of the workplace. Thirty-seven of the 84 workers had stopped working at ITO before the last year of the present surveillance period (table 1, 'former workers in 2010'). In a previous report, ${ }^{3}$ we confirmed that all variables, including functional, radiological and biochemical parameters, were qualitatively similar between the formerly and currently employed indium-exposed workers with similar exposure periods; therefore, we combined the data for the former and current workers to analyse the time course of the disease. All subjects provided their informed consent prior to initiation of the analysis in this study. In both the figures and tables, personal information has been carefully anonymised.

Because the severity of lung disorder caused by indium inhalation is correlated with the sIn concentration, ${ }^{35}$ we classified all workers into four groups according to their sIn concentrations measured in the first year: Group 1, <3.7; Group 2, 3.7-9.9; Group 3, 10.0-22.4; Group 4, >22.5 ng/mL. No differences were observed between the four groups in terms of age, prevalence of smokers or smoking indices. We analysed chronological changes in the variables among all participants and each of the four groups.

\section{Improvements in the work environment and practice}

The ITO processing work environment has steadily improved in addition to the introduction of various protective devices for workers. As a result, the indium concentration in the air, as measured in accordance with the Working Environment Measurement Law established by the Japan Industrial Safety and Health Association (JISHA), has gradually decreased (figure 2), and it can be assumed that the new indium burden has decreased for the study population as a whole.

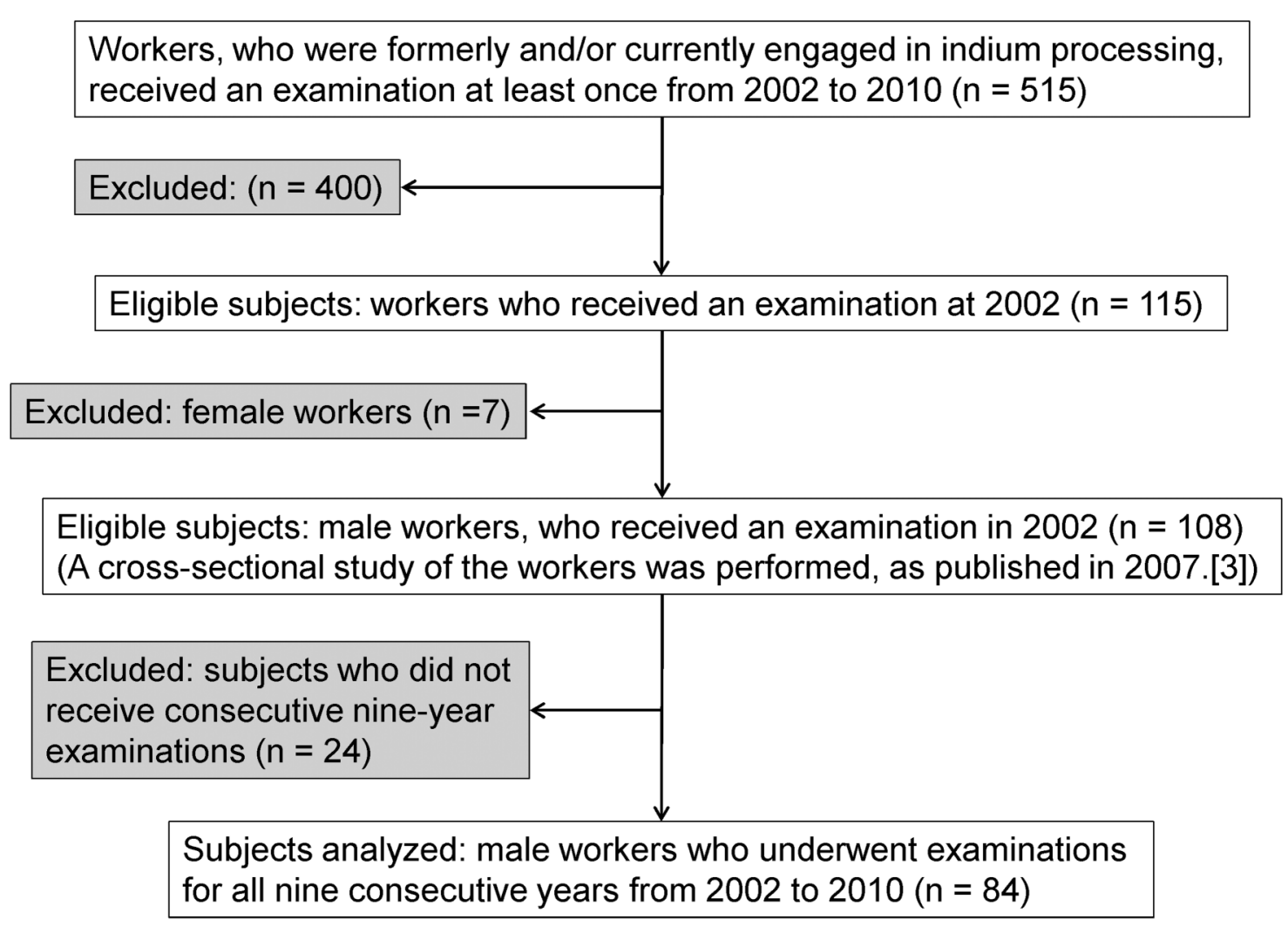

Figure 1 Study flowchart. 
Table 1 Worker characteristics

\begin{tabular}{|c|c|c|c|c|c|}
\hline & All & Group 1 & Group 2 & Group 3 & Group 4 \\
\hline Serum indium concentration, $\mathrm{ng} / \mathrm{mL}$ & $0.2-126.7$ & $0.2-3.6$ & $3.7-9.9$ & $10.0-22.4$ & $22.5-126.7$ \\
\hline Subjects, $\mathrm{n}$ & 84 & 21 & 21 & 21 & 21 \\
\hline Former workers, $\mathrm{n}$ (in 2010) & 37 & 6 & 5 & 9 & 17 \\
\hline Former workers, $\mathrm{n}$ (in 2002) & 21 & 6 & 4 & 3 & 8 \\
\hline Age, year (in 2002) & $33.9 \pm 7.9$ & $35.9 \pm 8.4$ & $33.7 \pm 7.8$ & $32.8 \pm 9.1$ & $33.3 \pm 6.1$ \\
\hline Exposure period, years (in 2010) & $9.7 \pm 4.8$ & $7.0 \pm 4.5$ & $9.0 \pm 4.2$ & $11.6 \pm 4.3$ & $11.5 \pm 4.5$ \\
\hline Period from first exposure, years (in 2010) & $14.5 \pm 4.8$ & $12.7 \pm 5.4$ & $13.1 \pm 4.0$ & $14.4 \pm 4.1$ & $17.7 \pm 3.8$ \\
\hline Period from last exposure, years* (in 2010) & $3.9 \pm 5.3$ & $4.1 \pm 6.4$ & $3.3 \pm 6.2$ & $2.2 \pm 3.3$ & $5.9 \pm 3.9$ \\
\hline Smokers (current and/or former), $\mathrm{n}$ (in 2010) & 68 & 18 & 19 & 16 & 15 \\
\hline Smokers in subjects, \% (in 2010) & 81.0 & 85.7 & 90.5 & 76.2 & 71.4 \\
\hline Smoking history, pack-years (in 2010) & $14.4 \pm 9.0$ & $16.2 \pm 9.0$ & $15.7 \pm 9.1$ & $12.0 \pm 8.7$ & $13.4 \pm 8.6$ \\
\hline
\end{tabular}

\section{Measurements}

The measurements obtained were those previously described. ${ }^{3}$ Specifically, the subjects' respiratory signs (rales, clubbed finger, cyanotic changes on finger nails) and symptoms (cough, sputum and exertional dyspnoea) were examined according to the Japanese Pneumoconiosis Questionnaire. ${ }^{15}$

Measurement of the serum KL-6, SP-D and indium concentrations The serum KL-6 and surfactant protein D (SP-D) levels were measured using an electrochemiluminescence immunoassay (Special Reference Laboratory, Tokyo, Japan), and the sIn concentration was measured by JISHA using inductively coupled plasma mass spectrometry (ICP-MS; Agilent Technologies, Santa Clara, California, USA). The serum SP-D levels had been measured since 2004. The KL-6 levels obtained in 2010 were omitted due to a temporal mechanical failure to measure KL-6 during this year. The SP-D levels obtained in 2008-2009 were excluded from the analysis due to a change in the measuring kit for SP-D in 2008-2009.

\section{Pulmonary function tests}

Spirometry parameters, including the vital capacity (VC), $\mathrm{FEV}_{1}$, $\mathrm{DL}_{\mathrm{CO}}$ and total lung capacity (TLC), were obtained using previously described methods. ${ }^{3}$

HRCT of the lungs

Three levels (bilateral upper, middle and lower lung fields) of the lung on HRCT were selected to score interstitial and emphysematous changes. The scoring method, a modified form of the Japanese Respiratory Society guidelines for the diagnosis and treatment of COPD, ${ }^{16}$ was utilised as previously described. ${ }^{3}$ Images of the three levels of the lungs were visually scored bilaterally for interstitial (ground-glass opacity or reticulonodular shadows) and emphysematous changes (low attenuation areas or

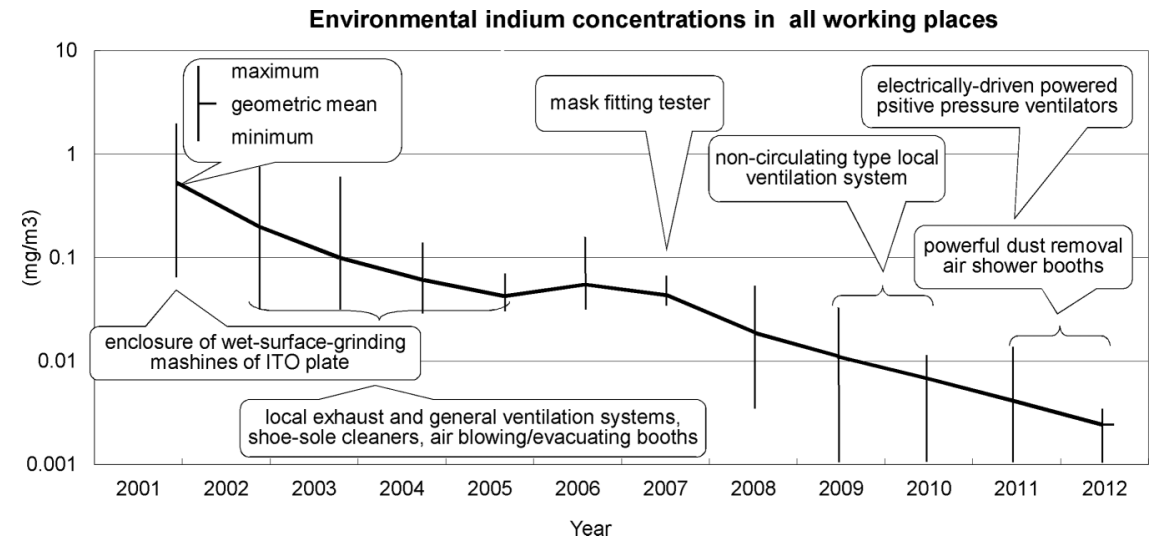

Figure 2 The annual trends in the environmental indium concentrations in all workplaces where indium compounds are handled from 2001 to 2012 (2001, six workplaces; 2002-2012, eight workplaces). The environmental indium concentrations were presented as the first evaluation values which were measured in all indium working places (the first evaluation value; the 95-percentile highest value estimated from the distribution curve of indium concentrations which are sampled in at least six sampling points in a work place, according to the Work Environment Measurement Law in Japan; the first evaluation values were calculated in each working place, and the geometric mean and maximum/minimum values were presented). The wet-surface-grinding machines of indium-tin oxide (ITO) plate were enclosed in boxes in 2001. The usage of the dust respirator with a $99.9 \%$ trap rate was strictly mandatory thereafter. From 2002 to 2005 , local exhaust and general ventilation systems were replaced by more powerful types. The shoe-sole cleaners and air blowing/evacuating booths were installed at the entrance/exit of the working rooms in order to eliminate the contamination of particles containing indium which attached to the shoes and working clothes. In 2007, a mask fitting tester was employed to confirm the proper use of the ventilators. In 2009 and 2010, the non-circulating type local ventilation system was located, which exhausted the room air and supplied newly cleaned-up air. In 2011, the dust respirators were replaced by electrically driven positive pressure ventilators, of which the trap rate was 99.97\%. The air blowing/evacuation booths were upgraded to blow more powerfully in 2011 and 2012 . 
bullae) on a scale of $0-5$ by the same two pulmonologists independently. The total score of six images was obtained, and the average total score for the two readers was adopted as the individual's score for interstitial and emphysematous changes, respectively. HRCT scoring was performed at the end of the study with the films displayed sequentially, while subject identifiers were blinded to the readers. The HRCT scores for 2008 were unavailable because low-dose helical CT was conducted rather than HRCT to explore the existence of lung cancer in that year.

The difference in scores between the two examiners was either 0 or 1 in $64 \%$ and $90 \%$ for interstitial and emphysematous changes, respectively (Spearman's rank correlations 0.75 and 0.77 , respectively). If the difference in score was equal to or greater than two, then the two examiners discussed and reevaluated the scores independently, and the average of the newly reevaluated scores was adopted.

\section{Statistical analysis}

For continuous variables, the data distribution was examined and appropriate transformation was performed to obtain an approximately normal distribution prior to the analysis. For assessing intra-individual changes of the parameters in each subject during the observation years, a single linear regression model was applied with the examination parameter as a dependent variable and study year as an independent variable, and the slope of the former against the latter was employed as an average annual change (AAC) of the examination parameter. Dunnett's test or Steel's test was applied for testing the AAC between the lowest sIn group (reference group) and those of the other three groups. To test the year-by-year trend of the parameters, a single or multiple linear regression model was applied for continuous variables and the Cochran-Armitage trend test was used for the prevalence data. Holm's method was used to avoid overestimation by multiple comparisons. The interaction between annual intra-individual time point variations and intergroup variations was tested using an ANOVA for repeated measurements with an adjusted degree of freedom for the $\mathrm{F}$ value using the Huynh-Feldt correction. All statistical analyses were performed using the JMP V.10.0.2/11.0.0 software programme (SAS Institute). Statistical significance was considered to exist at $\mathrm{p}<0.05$.

\section{RESULTS}

\section{Respiratory symptoms}

No obvious trends were observed regarding changes in the respiratory symptoms. None of the workers complained of dyspnoea in 2002, whereas three workers in Group 4 complained of exertional dyspnoea in 2010 (two of the three workers were non-smokers and the remaining one was an ex-smoker). Their sIn values in 2002 were 126.76, 98.76 and $40.42 \mathrm{ng} / \mathrm{mL}$, respectively, and the former two had the highest sIn levels among the 84 subjects. Moreover, their interstitial change scores on HRCT were among the largest $(14.5,17.0,17.0$, respectively) and they also exhibited emphysematous changes in 2002. Accordingly, the sIn level and interstitial HRCT changes may be surrogate makers of symptomatic deterioration.

\section{sln level}

The mean sIn level tended to decrease gradually from 2002 overall, as well as in each group (table 2). The individual slope of sIn over time was negative in 77 workers, regardless of whether they left or stayed in indium sections.

\section{Estimated biological half-life of sin}

The biological half-life of sIn was calculated from the rate of reduction in the sIn concentration obtained by plotting the sIn concentration against the number of years since quitting work in indium processing. The data for the participants who had quit handling indium for more than 3 years were analysed $(n=34) ; 26$ workers were current or former smokers and eight were never-smokers. The median (IQR) half-life was 8.09 years (5.91-13.79), which did not correlate with the subjects' smoking history. To elucidate the effects of the indium burden on the biological half-life of sIn, we divided the workers into

Table 2 Chronological changes in the sln, KL-6 and SP-D levels

\begin{tabular}{|c|c|c|c|c|c|c|c|c|c|c|}
\hline & 2002 & 2003 & 2004 & 2005 & 2006 & 2007 & 2008 & 2009 & 2010 & $p$ for trend \\
\hline \multicolumn{11}{|l|}{$\sin$} \\
\hline All & $8.6(4.2)$ & $7.5(4.7)$ & $8.0(4.3)$ & $6.5(5.1)$ & $4.4(7.2)$ & $5.6(5.4)$ & $5.8(4.8)$ & $4.9(5.2)$ & $4.9(4.9)$ & 0.0019 \\
\hline Gr 1 & $1.2(2.4)$ & $1.0(3.8)$ & $1.2(3.3)$ & $0.8(3.9)$ & $0.3(4.7)$ & $0.6(3.9)$ & $0.7(2.9)$ & $0.6(4.1)$ & $0.6(3.4)$ & 0.0071 \\
\hline Gr 2 & $6.0(1.3)$ & $5.3(1.3)$ & $5.7(1.4)$ & $5.0(1.4)$ & $3.4(2.1)$ & $4.3(1.5)$ & $4.3(1.5)$ & $4.2(1.5)$ & $4.0(1.6)$ & $<0.0001$ \\
\hline Gr 3 & $16.2(1.3)$ & $14.1(1.3)$ & $14.3(1.4)$ & $12.7(1.5)$ & $11.1(1.5)$ & $11.8(1.6)$ & $11.1(1.5)$ & $8.8(1.5)$ & $8.8(1.5)$ & $<0.0001$ \\
\hline Gr 4 & $46.4(1.6)$ & $41.4(1.7)$ & $41.0(1.7)$ & $37.8(1.7)$ & $33.5(1.7)$ & $32.8(1.8)$ & $33.6(1.7)$ & $28.8(1.7)$ & $27.9(1.7)$ & $<0.0001$ \\
\hline \multicolumn{11}{|l|}{ KL-6 } \\
\hline All & $448.0(2.1)$ & $404.2(2.0)$ & $376.2(1.9)$ & $348.0(1.9)$ & $352.2(1.8)$ & 297.7 (1.8) & $289.9(1.7)$ & $275.0(1.8)$ & NA & $<0.0001$ \\
\hline Gr 1 & $243.8(1.5)$ & $222.4(1.4)$ & $220.0(1.4)$ & 227.1 (1.5) & $228.8(1.4)$ & $205.8(1.4)$ & $198.2(1.4)$ & 187.9 (1.4) & NA & 0.0102 \\
\hline Gr 2 & 383.8 (1.7) & $345.4(1.7)$ & $327.3(1.7)$ & 293.8 (1.6) & 316.7 (1.6) & $266.3(1.5)$ & $253.7(1.4)$ & $258.3(1.6)$ & NA & 0.0002 \\
\hline Gr 3 & $452.2(2.0)$ & $418.0(1.7)$ & $360.8(1.7)$ & $328.2(1.7)$ & 339.9 (1.7) & $259.3(1.4)$ & $265.8(1.5)$ & 239.7 (1.6) & NA & $<0.0001$ \\
\hline Gr 4 & $952.2(1.7)$ & 831.7 (1.7) & 770.8 (1.7) & $669.3(1.7)$ & $624.4(1.8)$ & $552.6(1.8)$ & 528.5 (1.7) & $491.7(1.8)$ & NA & $<0.0001$ \\
\hline \multicolumn{11}{|l|}{ SP-D } \\
\hline All & & & 75.0 (1.8) & $65.7(1.7)$ & $61.9(1.9)$ & $53.5(1.8)$ & NA & NA & $39.8(2.3)$ & $<0.0001$ \\
\hline Gr 1 & & & $57.1(1.7)$ & $51.9(1.6)$ & $49.4(1.9)$ & $46.7(1.8)$ & NA & NA & $34.4(2.2)$ & 0.0049 \\
\hline Gr 2 & & & $57.9(1.6)$ & $52.1(1.5)$ & $48.2(1.7)$ & $42.7(1.8)$ & NA & NA & $30.2(2.2)$ & 0.0001 \\
\hline Gr 3 & & & $73.5(1.7)$ & $64.9(1.6)$ & $57.8(1.8)$ & $47.8(1.6)$ & NA & NA & $33.7(2.2)$ & $<0.0001$ \\
\hline Gr 4 & & & 130.2 (1.6) & $106.3(1.7)$ & $106.4(1.6)$ & $85.7(1.7)$ & NA & NA & $71.3(1.9)$ & 0.0002 \\
\hline
\end{tabular}

Data are expressed as geometric mean (geometric SD).

Gr 1, sln $<3.7 \mathrm{ng} / \mathrm{mL} ;$ Gr 2, $3.7 \mathrm{ng} / \mathrm{m}<\sin <9.9 \mathrm{ng} / \mathrm{mL} ; \mathrm{Gr} \mathrm{3,} 10.0 \mathrm{ng} / \mathrm{mL}<\sin <22.4 \mathrm{ng} / \mathrm{mL} ; \mathrm{Gr} 4,22.5 \mathrm{ng} / \mathrm{mL}<\sin$.

${ }^{*}$ Test for chronological trend by applying single regression model.

Gr, group; NA, not available; sln, serum indium concentration; SP-D, surfactant protein D. 
three groups according to the sIn concentrations measured just before stopping indium processing: $<3.0 \quad(n=6), 3.1-10.0$ $(\mathrm{n}=7)$ and $>10.0 \mathrm{ng} / \mathrm{mL}(\mathrm{n}=21)$. The median half-life in the $>10.0 \mathrm{ng} / \mathrm{ml}$ group was 8.95 years (7.49-12.21), which was longer than those observed in the groups with lower sIn concentrations (5.80 and 6.63, respectively).

\section{Serum KL-6 and SP-D}

The mean serum KL-6 levels tended to decrease in 2002-2009 overall, as well as in each group (table 2). The mean serum SP-D levels also tended to decrease significantly from 2004 to 2010 overall (table 2). Additionally, the prevalence of workers with an abnormally elevated KL-6 or SP-D level decreased (table 3).

\section{Radiological findings}

During the nine-year study period, the interstitial HRCT score decreased overall but tended to remain stable during the latter half of the surveillance (figure $3 \mathrm{~A}$ and see online supplementary material). Conversely, the emphysematous lesions on HRCT showed continuous progression in the highest sIn group (figure 3B and see online supplementary material). No differences in the AACs in the emphysematous scores were observed among current, past and never-smokers, even in the highest sIn group (data not shown). No lung cancer was identified according to low-dose helical CT.

\section{Pulmonary function tests}

No consistent changes were observed in $\% \mathrm{FEV}_{1}$ and \%TLC in any group (table 4 ). Conversely, $\% \mathrm{VC}$ in all groups and $\% \mathrm{DL}_{\mathrm{CO}}$ in Group 3 slightly increased. The $\mathrm{FEV}_{1} / \mathrm{FVC}$ in Groups 2-4 decreased over time. The average annual decline in the $\mathrm{FEV}_{1} /$ FVC was significantly greater in Group 4 compared with Group 1 (see online supplementary material). The \%VC, \% $\mathrm{FEV}_{1}, \% \mathrm{DLco}$ and \%TLC in Group 4 were lower than those in the other groups during most years. There were no differences between the smokers and non-smokers regarding the relationship between the smoking history and annual changes in \% $\mathrm{VC}$, $\% \mathrm{FEV}_{1}, \% \mathrm{DL}_{\mathrm{CO}}$ and \%TLC (data not shown).

\section{DISCUSSION}

We conducted a 9-year comprehensive longitudinal surveillance of indium lung after cessation or decrease in the exposure to inhaled indium compounds. The sIn, KL-6, SP-D and the degree of interstitial lesions on HRCT decreased with the reduction in exposure to indium; however, in the workers with high sIn concentrations, emphysematous lesions increased over time. This surveillance is the longest study available to date and was performed in a single plant where a previous cross-sectional study was conducted. ${ }^{3}$ The findings of the present study reinforce the conclusions of a recent 5-year follow-up study conducted in 11 factories. ${ }^{17}$

Improvements in the work environment with the obligatory use of room ventilators, mask fitting testers, and air shower booths have been implemented annually since the first reported case of indium-related lung disease (figure 2). Workers were transferred to non-indium sections according to the results of annual examinations based on the predetermined criteria ('Study design and subjects' in the Materials and Methods section). As a result, the average sIn concentration in the present surveillance decreased annually in workers with high and low sIn. These results suggest that it is possible to eliminate deposited indium in the body, albeit very slowly, with continuous efforts to reduce the level of inhaled indium.

The serum KL-6 and SP-D levels have been reported to be useful markers of the disease activity in patients with indium lung $^{3}$ and interstitial pneumonia. ${ }^{18}{ }^{19}$ In this study, the serum KL-6 and SP-D levels declined over the observation period, and the prevalence of workers with elevated serum KL-6 or SP-D levels declined significantly, despite that the prevalence of sIn $>3 \mathrm{ng} / \mathrm{mL}$ remained $66.7 \%$ in 2010 from $76.2 \%$ in 2002 ( $\mathrm{sIn}$ value $<3 \mathrm{ng} / \mathrm{mL}$ is a potential cut-off value for preventing interstitial lung disease from indium exposure ${ }^{5}$ ). Although indium-induced interstitial pneumonia has been suggested to be partly attenuated by the reduction of indium exposure, the clearance of indium appears to require longer periods of time.

The role of smoking in the pathogenesis of indium lung, especially emphysematous changes, must be addressed. There were no differences among smokers, ex-smokers and nonsmokers in the progression rate of the emphysematous score even in the highest sIn group, suggesting that the emphysematous change was not related to the smoking history, but rather caused by indium compounds. This may be explained by the fact that the indium workers were relatively young and the smoking duration was short in this study (table 1). The small

Table 3 The prevalence (\%) of workers with abnormally elevated KL-6 and SP-D levels

\begin{tabular}{|c|c|c|c|c|c|c|c|c|c|c|}
\hline & 2002 & 2003 & 2004 & 2005 & 2006 & 2007 & 2008 & 2009 & 2010 & $p$ for trend* \\
\hline \multicolumn{11}{|l|}{ KL-6 } \\
\hline All & 39.3 & 35.7 & 32.1 & 27.4 & 25.0 & 15.5 & 14.3 & 13.1 & NA & $<0.0001$ \\
\hline Gr 1 & 4.8 & 0 & 0 & 9.5 & 4.8 & 0 & 0 & 0 & NA & 0.0173 \\
\hline Gr 2 & 19.0 & 19.0 & 14.3 & 9.5 & 9.5 & 9.5 & 4.8 & 4.8 & NA & 0.0007 \\
\hline Gr 3 & 47.6 & 38.1 & 28.6 & 19.0 & 19.0 & 4.8 & 9.5 & 9.5 & NA & $<0.0001$ \\
\hline Gr 4 & 85.7 & 85.7 & 85.7 & 71.4 & 66.7 & 47.6 & 42.9 & 38.1 & NA & 0.0003 \\
\hline \multicolumn{11}{|l|}{ SP-D } \\
\hline All & & & 26.2 & 17.9 & 19 & 11.9 & NA & NA & 7.1 & $<0.0001$ \\
\hline Gr 1 & & & 9.5 & 0 & 0 & 4.8 & NA & NA & 0 & 0.0137 \\
\hline Gr 2 & & & 9.5 & 0 & 4.8 & 0 & NA & NA & 0 & 0.0007 \\
\hline Gr 3 & & & 14.3 & 9.5 & 9.5 & 4.8 & NA & NA & 4.8 & 0.0001 \\
\hline Gr 4 & & & 71.4 & 61.9 & 61.9 & 38.1 & NA & NA & 23.8 & 0.0001 \\
\hline
\end{tabular}

All data are expressed as \%. The data for KL-6 in 2010 and SP-D in 2008 and 2009 were not available due to technical reasons.

Gr 1, sln<3.7 ng/mL; Gr 2, $3.7 \mathrm{ng} / \mathrm{mL}<\mathrm{s} / \mathrm{n}<9.9 \mathrm{ng} / \mathrm{mL} ; \mathrm{Gr} 3,10.0 \mathrm{ng} / \mathrm{mL}<\mathrm{s} \ln <22.4 \mathrm{ng} / \mathrm{mL} ; \mathrm{Gr} 4,22.5 \mathrm{ng} / \mathrm{mL}<$ sln. The cut-off values of abnormal KL-6 and SP-D levels were $>500 \mathrm{U} / \mathrm{mL}$ and $>110 \mathrm{ng} / \mathrm{mL}$, respectively.

* The Cochran-Armitage trend test was used to test the significance of annual trends among the parameters.

Gr, group; NA, not available; sln, serum indium level; SP-D, surfactant protein D. 


\section{Chronological changes in HRCT}
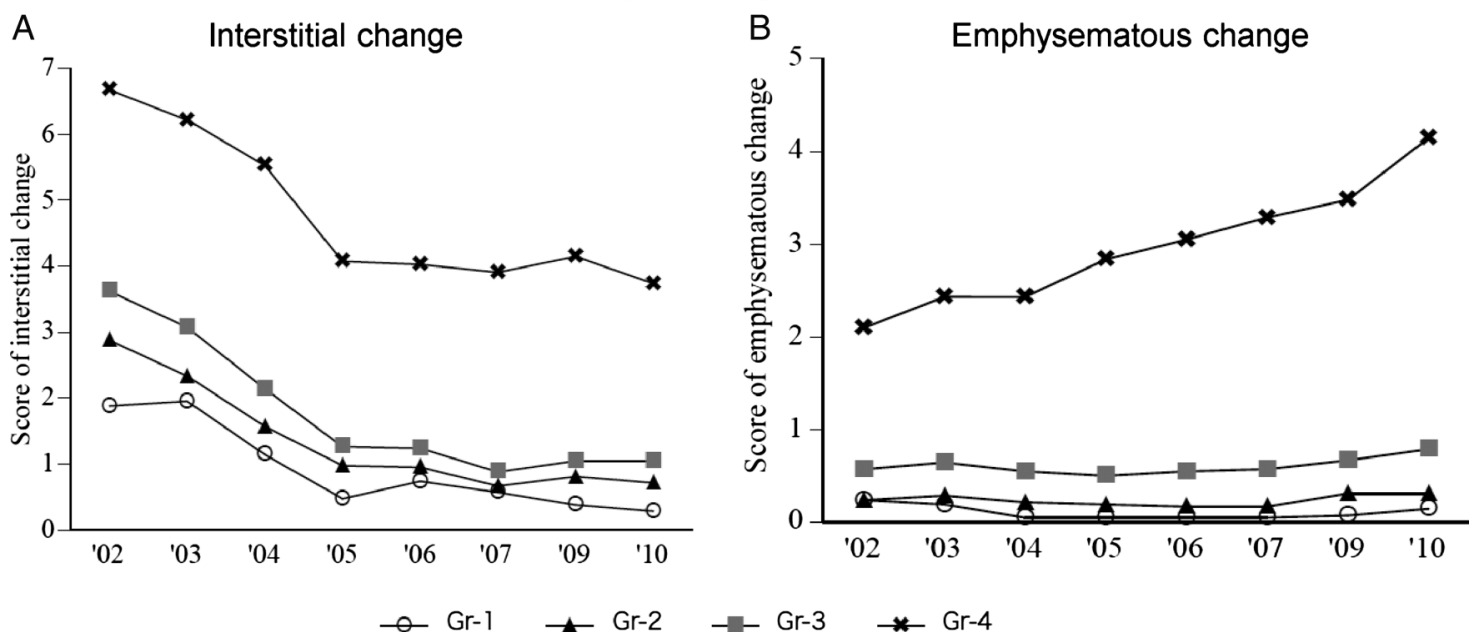

- Gr-1 - $-\mathrm{Gr}-3 \quad$ * $-\mathrm{Gr}-4$

Figure 3 Chronological changes in (A) interstitial and (B) emphysematous lesion score on high-resolution CT (HRCT) at four levels of the serum indium (s/n) concentration. The data $(A$ and $B$ ) are presented as the mean. Group (Gr) 1, sln $<3.7 \mathrm{ng} / \mathrm{mL} ; \mathrm{Gr} 2,3.7 \mathrm{ng} / \mathrm{mL}<\mathrm{s} / \mathrm{n}<9.9 \mathrm{ng} / \mathrm{mL} ; \mathrm{Gr} 3$, $10.0 \mathrm{ng} / \mathrm{mL}<\sin <22.4 \mathrm{ng} / \mathrm{mL} ; \mathrm{Gr} 4,22.5 \mathrm{ng} / \mathrm{mL}<\sin$.

Table 4 Chronological changes of the pulmonary function tests

\begin{tabular}{|c|c|c|c|c|c|c|c|c|c|c|}
\hline & \multicolumn{10}{|c|}{ Pulmonary function test by year } \\
\hline & 2002 & 2003 & 2004 & 2005 & 2006 & 2007 & 2008 & 2009 & 2010 & $p$ for trend* \\
\hline \multicolumn{11}{|c|}{$\%$ VC (\%) } \\
\hline All & $98.3 \pm 11.4$ & $100.2 \pm 11.7$ & $98.4 \pm 12.1$ & $101.0 \pm 11.9$ & $100.9 \pm 11.5$ & $102.6 \pm 11.5$ & $105.0 \pm 11.5$ & $106.9 \pm 12.3$ & $102.5 \pm 11.6$ & $<0.001$ \\
\hline Gr 1 & $102.1 \pm 9.7$ & $104.2 \pm 9.1$ & $102.7 \pm 10.1$ & $105.1 \pm 10.5$ & $104.6 \pm 10.0$ & $105.8 \pm 9.7$ & $107.2 \pm 9.5$ & $109.1 \pm 11.2$ & $104.8 \pm 8.7$ & 0.0327 \\
\hline Gr 2 & $99.7 \pm 12.1$ & $101.6 \pm 11.8$ & $99.9 \pm 11.6$ & $103.2 \pm 11.9$ & $102.9 \pm 11.5$ & $104.6 \pm 11.1$ & $107.0 \pm 13.0$ & $108.9 \pm 13.0$ & $103.6 \pm 12.7$ & 0.0103 \\
\hline Gr 3 & $98.6 \pm 11.7$ & $101.1 \pm 12.3$ & $99.8 \pm 13.0$ & $101.3 \pm 12.6$ & $101.2 \pm 11.4$ & $103.7 \pm 12.6$ & $106.4 \pm 11.6$ & $107.1 \pm 12.4$ & $104.6 \pm 11.8$ & 0.0053 \\
\hline Gr 4 & $92.6 \pm 10.7$ & $93.7 \pm 11.1$ & $91.2 \pm 11.0$ & $94.6 \pm 10.5$ & $95.1 \pm 11.3$ & $96.5 \pm 10.9$ & $99.5 \pm 10.6$ & $102.5 \pm 12.1$ & $96.9 \pm 11.5$ & 0.0010 \\
\hline \multicolumn{11}{|c|}{$\% \mathrm{FEV}_{1}(\%)$} \\
\hline All & $92.7 \pm 10.4$ & $93.3 \pm 12.0$ & $92.1 \pm 12.7$ & $92.0 \pm 12.4$ & $91.3 \pm 12.5$ & $92.9 \pm 11.4$ & $94.9 \pm 12.0$ & $94.6 \pm 16.3$ & $92.8 \pm 12.9$ & 0.3132 \\
\hline Gr 1 & $96.1 \pm 5.9$ & $96.2 \pm 6.7$ & $96.5 \pm 8.2$ & $95.5 \pm 9.3$ & $95.3 \pm 5.9$ & $96.9 \pm 8.0$ & $97.7 \pm 9.5$ & $95.7 \pm 23.4$ & $96.9 \pm 11.0$ & 0.7717 \\
\hline Gr 2 & $94.0 \pm 9.9$ & $95.7 \pm 11.2$ & $93.4 \pm 10.4$ & $93.9 \pm 10.8$ & $91.9 \pm 9.8$ & $93.7 \pm 10.1$ & $96.4 \pm 11.5$ & $96.3 \pm 12.0$ & $92.4 \pm 11.2$ & 0.9350 \\
\hline Gr 3 & $95.1 \pm 10.6$ & $96.0 \pm 11.9$ & $95.3 \pm 12.2$ & $94.9 \pm 11.5$ & $94.2 \pm 12.3$ & $96.5 \pm 10.7$ & $98.5 \pm 10.7$ & $98.8 \pm 12.2$ & $96.3 \pm 12.3$ & 0.2819 \\
\hline Gr 4 & $85.6 \pm 11.5$ & $85.5 \pm 14.1$ & $83.1 \pm 14.9$ & $83.9 \pm 14.4$ & $83.6 \pm 15.1$ & $84.5 \pm 12.5$ & $87.1 \pm 13.2$ & $87.6 \pm 13.5$ & $85.6 \pm 14.4$ & 0.5114 \\
\hline \multicolumn{11}{|c|}{$\mathrm{FEV}_{1} / \mathrm{FVC}$ ratio $(\%)$} \\
\hline All & $82.8 \pm 7.0$ & $81.5 \pm 7.1$ & $81.2 \pm 7.6$ & $80.1 \pm 8.6$ & $79.9 \pm 9.0$ & $79.8 \pm 7.9$ & $78.8 \pm 8.1$ & $78.8 \pm 8.3$ & $78.6 \pm 8.2$ & $<0.001$ \\
\hline Gr 1 & $82.4 \pm 5.8$ & $80.9 \pm 5.8$ & $81.1 \pm 6.2$ & $79.6 \pm 6.7$ & $79.2 \pm 6.1$ & $79.6 \pm 6.1$ & $78.7 \pm 6.3$ & $79.4 \pm 6.6$ & $79.8 \pm 6.7$ & 0.0646 \\
\hline Gr 2 & $82.4 \pm 6.1$ & $81.6 \pm 6.1$ & $81.3 \pm 6.7$ & $79.8 \pm 7.3$ & $79.8 \pm 6.2$ & $80.4 \pm 8.6$ & $78.7 \pm 7.1$ & $78.7 \pm 6.9$ & $77.5 \pm 6.6$ & 0.0041 \\
\hline Gr 3 & $84.9 \pm 7.1$ & $83.8 \pm 6.4$ & $83.3 \pm 6.7$ & $82.5 \pm 7.9$ & $82.4 \pm 7.8$ & $81.9 \pm 7.8$ & $81.6 \pm 7.9$ & $81.9 \pm 7.5$ & $80.6 \pm 7.9$ & 0.0279 \\
\hline Gr 4 & $81.3 \pm 8.9$ & $79.7 \pm 9.3$ & $79.2 \pm 10.0$ & $78.4 \pm 11.7$ & $78.3 \pm 13.4$ & $77.1 \pm 9.3$ & $76.1 \pm 10.3$ & $75.3 \pm 10.7$ & $76.5 \pm 10.6$ & 0.0239 \\
\hline \multicolumn{11}{|c|}{$\% \mathrm{DL}_{\mathrm{CO}}(\%)$} \\
\hline All & $115.4 \pm 16.5$ & $110.1 \pm 18.2$ & $105.2 \pm 17.7$ & $104.1 \pm 17.0$ & $109.1 \pm 18.0$ & $113.0 \pm 19.1$ & $119.3 \pm 19.8$ & $112.3 \pm 18.6$ & $114.0 \pm 19.9$ & 0.0159 \\
\hline Gr 1 & $124.4 \pm 14.0$ & $117.6 \pm 15.0$ & $115.6 \pm 12.7$ & $113.0 \pm 12.8$ & $119.6 \pm 18.3$ & $121.6 \pm 15.6$ & $128.0 \pm 17.5$ & $121.0 \pm 14.2$ & $125.9 \pm 13.9$ & 0.6671 \\
\hline Gr 2 & $115.2 \pm 11.5$ & $110.1 \pm 14.1$ & $105.6 \pm 11.6$ & $105.0 \pm 11.7$ & $108.7 \pm 14.6$ & $112.6 \pm 12.3$ & $116.7 \pm 14.9$ & $112.7 \pm 15.8$ & $112.9 \pm 17.2$ & 0.9362 \\
\hline Gr 3 & $118.6 \pm 10.0$ & $114.0 \pm 14.3$ & $107.4 \pm 10.2$ & $106.5 \pm 14.6$ & $109.5 \pm 13.6$ & $118.1 \pm 13.6$ & $124.8 \pm 17.3$ & $115.4 \pm 13.0$ & $116.5 \pm 14.0$ & 0.0035 \\
\hline Gr 4 & $103.3 \pm 20.4$ & $98.6 \pm 22.9$ & $92.0 \pm 24.4$ & $92.1 \pm 20.9$ & $98.6 \pm 19.5$ & $99.7 \pm 25.3$ & $107.8 \pm 23.3$ & $100.1 \pm 23.7$ & $100.7 \pm 24.9$ & 0.0572 \\
\hline \multicolumn{11}{|c|}{$\% \operatorname{TLC}(\%)$} \\
\hline All & $109.2 \pm 11.8$ & $106.6 \pm 12.6$ & $104.8 \pm 12.9$ & $104.4 \pm 12.2$ & $105.5 \pm 12.3$ & $110.0 \pm 12.9$ & $112.4 \pm 13.2$ & $105.8 \pm 12.3$ & $105.3 \pm 12.3$ & 0.7575 \\
\hline Gr 1 & $113.2 \pm 7.5$ & $110.3 \pm 9.2$ & $109.5 \pm 10.4$ & $108.1 \pm 9.1$ & $109.4 \pm 10.8$ & $113.5 \pm 10.7$ & $116.5 \pm 11.6$ & $110.0 \pm 10.5$ & $109.7 \pm 10.8$ & 0.8201 \\
\hline Gr 2 & $110.7 \pm 12.6$ & $109.6 \pm 12.1$ & $106.5 \pm 13.2$ & $106.0 \pm 12.3$ & $107.1 \pm 12.8$ & $114.1 \pm 14.0$ & $113.3 \pm 13.7$ & $106.6 \pm 13.1$ & $105.8 \pm 13.0$ & 0.7487 \\
\hline Gr 3 & $109.1 \pm 12.9$ & $107.1 \pm 13.6$ & $105.2 \pm 12.9$ & $105.2 \pm 13.4$ & $105.5 \pm 12.4$ & $109.8 \pm 11.9$ & $113.7 \pm 12.7$ & $105.2 \pm 11.9$ & $105.2 \pm 11.6$ & 0.9982 \\
\hline Gr 4 & $103.5 \pm 12.3$ & $99.2 \pm 12.5$ & $97.9 \pm 12.8$ & $98.2 \pm 12.2$ & $100.0 \pm 12.0$ & $102.5 \pm 12.3$ & $106.1 \pm 13.3$ & $101.4 \pm 12.9$ & $100.8 \pm 13.0$ & 0.4424 \\
\hline
\end{tabular}


sample size may also be a limitation in clarifying the role of smoking in indium lung.

The validity of our scoring method using HRCT has been discussed previously. ${ }^{3}$ The interstitial changes on HRCT decreased during the first years of the study period and then stabilised for the remaining years. Cummings $e t \mathrm{al}^{14}$ reported that pathologic reevaluation showed evidence of cholesterol clefts and proteinaceous material in the alveoli, consistent with alveolar proteinosis in most Japanese cases, which was originally reported as pulmonary fibrosis. We did not previously recognise, however, the interstitial changes (eg, ground-glass opacity) on HRCT may have partly reflected alveolar proteinosis and diminished over the first few years. This may be compatible with the hypothesis that partially reversible alveolar proteinosis is induced by the inhalation of indium compounds and pulmonary fibrosis occurs later in cases involving heavy indium deposition. Conversely, the emphysematous changes gradually worsened in the group with the highest sIn concentrations. The emphysematous progression of indium workers with high sIn levels were confirmed in the most recent cohort study. ${ }^{17}$ Regarding the progress of emphysematous lesion, the check-valve mechanism resulting from bronchiolar obstruction by cholesterol granuloma formation and fibrous changes with heavy deposition of indium compounds may have occurred. ${ }^{2} 6$

In the pulmonary function tests, the complicated trends observed (ie, decreased $\mathrm{FEV}_{1} / \mathrm{FVC}$ and slightly improved $\mathrm{VC}$ and $\mathrm{DL}_{\mathrm{CO}}$ ) in the high sIn groups may be attributed to the mixture of improved interstitial (eg, proteinosis) and worsened emphysematous changes. The presence of airway obstruction accompanied by the emphysematous changes may have affected the $\mathrm{FEV}_{1} /$ FVC, which is supported by the result that the decline of $\mathrm{FEV}_{1} /$ FVC in Group 4 was significantly larger than that of Group 1. In a recent study, Cummings et $a l^{20}$ reported that abnormalities in the pulmonary function are common in ITO processing workers, especially those with a high sIn concentration. Therefore, even though the present study did not reveal consistent changes in the $\mathrm{FEV}_{1}, \mathrm{DL}_{\mathrm{CO}}$ and TLC in indium workers, careful follow-up of the pulmonary function over a longer duration is required.

In this study, the estimated median sIn half-life of decay was 8.09 years; this value was longer in the workers with high sIn concentrations. We speculate that, in such workers, the pulmonary vasculature is more severely damaged and the blood flow to the lungs is limited, which subsequently prolongs the clearance of deposited indium. Similarly, the mucociliary transport system may be more severely damaged in workers with a heavy indium burden, which may also inhibit indium clearance. These remain to be explored together with the toxicity of indium on the alveolar macrophages. Nonetheless, there were no differences in the average rate of decrease of sIn, KL-6 and SP-D during the 9-year period between the current and former workers, suggesting that indium clearance is mainly determined by the amount of deposition rather than the post-exposure period. The laboratory data and HRCT changes in indium workers should be carefully followed with special attention to the evolution of indium-related lung disease, regardless of workplace transfer to non-indium-contaminated sections.

\section{CONCLUSIONS}

The results of the present study clarified several aspects of the recently recognised occupational lung disease 'indium lung'. Our findings suggest that (1) inhaled indium compounds in the lungs can only be eliminated very slowly following the reduction or cessation of exposure to indium, (2) the elimination of deposited indium is associated with decreases in the serum KL-6 and SP-D levels, and (3) although indium-induced interstitial changes are partially reversible, permanent emphysematous changes may occur under heavy exposure to indium compounds. Therefore, it is necessary to reduce exposure to indiumcontaining particles and detect indium-related lung disorders as early as possible.

Acknowledgements The authors thank Drs Hironori Masuko and Youhei Yatagai for their skilful contributions to the clinical evaluation of the acquired data.

Contributors $\mathrm{AA}, \mathrm{HN}$ and TC designed the study conception, conducted data analysis and prepared the manuscript. KO developed the data analysis and interpretation of such information. JT and KT contributed to writing the article or substantial involvement in its revision prior to submission.

Competing interests None declared.

Ethics approval The medical professional committee that oversees the health maintenance programme of the plant.

Provenance and peer review Not commissioned; externally peer reviewed.

\section{REFERENCES}

1 Omae K, Nakano M, Tanaka A, et al. Indium lung - case reports and epidemiology. Int Arch Occup Environ Health 2011;84:471-7.

2 Homma T, Ueno T, Sekizawa $\mathrm{K}$, et al. Interstitial pneumonia developed in a worker dealing with particles containing indium-tin oxide. J Occup Health 2003:45:137-9.

3 Chonan T, Taguchi 0, Omae K. Interstitial pulmonary disorders in indium-processing workers. Eur Respir J 2007:29:317-24.

4 Hamaguchi T, Omae K, Takebayashi T, et al. Exposure to hardly soluble indium compounds in ITO production and recycling plants is a new risk for interstitial lung damage. Occup Environ Med 2008;65:51-5.

5 Nakano M, Omae K, Tanaka A, et al. Causal relationship between indium compound inhalation and effects on the lungs. J Occup Health 2009:51:513-21.

6 Homma S, Miyamoto A, Sakamoto S, et al. Pulmonary fibrosis in an individual occupationally exposed to inhaled indium-tin oxide. Eur Respir J 2005:25:200-4.

7 Taguchi 0, Chonan T. [Three cases of indium lung]. Nihon Kokyuki Gakkai Zasshi 2006;44:532-6.

8 Nakano $M$, Kamata $H$, Saito $F$, et al. A case of indium lung disclosed in health check-up (Japanese). Occup Health J 2007;30:25-9.

9 Chonan T, Amata A, Nodera $\mathrm{H}$, et al. Interstitial pneumonia induced by inhalation of indium compounds. Jpn J Chest Dis 2010;69:851-5.

10 Takeuchi K. Pulmonary toxicity of indium (Japanese). Respiration (Kokyu) 2008;27:599-603

11 Tanaka R, Otsuka T, Nakamura $Y$, et al. A case of indium lung occurred by engaging in grinding metals (Japanese). Ann Japanese Respir Soc 2013;2 (Suppl):273.

12 Cummings KJ, Donat WE, Ettensohn DB, et al. Pulmonary alveolar proteinosis in workers at an indium processing facility. Am J Respir Crit Care Med 2010;181:458-64.

13 Xiao YL, Cai HR, Wang YH, et al. Pulmonary alveolar proteinosis in an indium-processing worker. Chin Med J (Engl) 2010;123:1347-50.

14 Cummings KJ, Nakano M, Omae K, et al. Indium lung disease. Chest 2012;141:1512-21.

15 The Labor Hygiene Section in the Safety Hygiene Department of Ministry of Labour. A handbook for examining pneumoconiosis. Japan Industrial Safety and Health Association, 1980:40-5.

16 The Japanese Respiratory Society. Guidelines for the diagnosis and treatment of COPD, 3rd edition. Tokyo: Medical Review Co Ltd, 2009.

17 Nakano M, Omae K, Uchida K, et al. Five-year cohort study: emphysematous progression of indium-exposed workers. Chest 2014;146:1166-75.

18 Kohno N, Kyoizumi S, Awaya Y, et al. New serum indicator of interstitial pneumonitis activity. Sialylated carbohydrate antigen KL-6. Chest 1989;96:68-73.

19 Honda $Y$, Kuroki Y, Matsuura E, et al. Pulmonary surfactant protein D in sera and bronchoalveolar lavage fluids. Am J Respir Crit Care Med 1995;152 (6 Pt 1):1860-6.

20 Cummings KJ, Suarthana E, Edwards $N$, et al. Serial evaluations at an indium-tin oxide production facility. Am J Ind Med 2013;56:300-7. 